\title{
Semi-Automated Rasch Analysis using In-plus-out-of-questionnaire Log-likelihood
}

\author{
Feri Wijayanto ${ }^{a, b}$, Karlien Mul ${ }^{c}$, Perry Groot $^{b}$, Baziel G.M. van Engelen ${ }^{c}$, Tom \\ Heskes $^{\mathrm{b}}$ \\ ${ }^{a}$ Department of Informatics, Universitas Islam Indonesia, Sleman, Indonesia \\ ${ }^{b}$ Institute for Computing and Information Sciences, Radboud University Nijmegen, The \\ Netherlands \\ ${ }^{c}$ Department of Neurology, Donders Institute for Brain, Cognition, and Behaviour, Radboud \\ University Medical Center, Nijmegen, The Netherlands
}

\begin{abstract}
Rasch analysis is a popular statistical tool for developing and validating instruments that aim to measure human performance, attitudes and perceptions. Despite the availability of various software packages, constructing a good instrument based on Rasch analysis is still considered to be a complex, labor-intensive task, requiring human expertise and bares the possibility results in different but equally suited instruments. In this paper, we propose a semi-automated method for Rasch analysis based on first principles that reduces the need for human input. To this end, we introduce a novel criterion, called in-plus-out-of-questionnaire log-likelihood (IPOQ-LL). On artificial datasets, we confirm that optimization of IPOQ-LL leads to the desired behavior in the case of multi-dimensional and inhomogeneous surveys. On three publicly available real-world datasets, our method leads to instruments that have, for all practical purposes, similar clinimetrical properties as those obtained by Rasch analysis experts through a manual procedure.

Keywords: Semi-automated Rasch analysis, Rasch model, 2PL model, Generalized partial credit model, Penalized JMLE
\end{abstract}

\section{Introduction}

Our theoretical understanding of the world often contains latent constructs or traits, such as "intelligence" and "quality of life" that cannot be assessed directly. In an attempt to infer these traits for individual people, thousands of scales have been developed that aim to measure a range of behaviors and experiences in sociology, psychology, and medicine. These scales are typically based on questionnaires that contain a number of items. An appropriate instrument is valid, i.e., indeed measures the latent construct it has been designed to measure, reliable, i.e., contains

*This is the pre-peer reviewed version of the following article: [43, which has been published in final form at https://doi.org/10.1111/bmsp.12218. This article may be used for non-commercial purposes in accordance with Wiley Terms and Conditions for Use of Self-Archived Versions. 
enough items to reliably estimate the scale for an individual person, and clinimetrically useful. For practical purposes, we prefer questionnaires with a limited number of representative items, to lessen the burden on the respondents and increase the reliability.

One of the modern ways to develop a linear-weighted, clinimetrically sound measurement instrument is Rasch analysis. The Rasch model can be used for creating measurements from categorical or ordinal data, as a function of the trade-off between the subject's abilities and the item difficulties. After conceptualization of the latent construct, a relatively large number of items are designed that are expected to relate to the trait under consideration, or an existing ordinal based measurement instrument can be used. This original survey is provided to a number of initial respondents, preferably a few hundred in order to provide a stable Rasch model [47]. Their responses are then used for subsequent analysis. In this procedure, items are evaluated based on various criteria inherent to the Rasch model [31, 32], such as item goodness-of-fit, unidimensionality, presence of differential item functioning (DIF) and local dependency [12, 14. The worst item or items are generally removed or remodelled within the model limits' possibilities (e.g. subset creation), after which the remaining items are reevaluated, until a clinimetrically optimal smaller itemset remains fulfilling all model's expectations.

This step-by-step procedure is typically done manually, by expert Rasch analysts, with the help of software packages such as Winstep, RUMM2030, ConQuest, or eRM [48]. This procedure can be relatively time consuming especially in cases of large or complex datasets. Furthermore, since the decisions which items to include partly rely on human judgments blended with clinical expertise, different experts often arrive at different but equally suited instruments.

The goal of this paper is to try and automate the process of turning an ordinal scale into a clinimetrically sound linear-weighted scale. We will show that our procedure naturally caters for typical Rasch criteria such as item goodness-of-fit, unidimensionality, and (to some extent) local dependency. We discuss extensions of our approach to also include differential item functioning, restoring of disordered thresholds, and possibly other criteria, but leave actual implementations of these ideas for future work. We will refer to our procedure as "semi-automated", to acknowledge that it only automates part of a full-blown Rasch analysis as well as to emphasize the need for sanity checks in any statistical analysis.

In order to deliver the idea, in the next section, we discuss our central model in the implementation, the generalized partial credit model (GPCM), its relation with other models and the idea to solve its estimation problem using regularization. Section 3 elaborates the vital part of our proposed method, the in-plus-out-of questionnaire log likelihood (IPOQ-LL), and argues for the method in comparison with the typical criteria of standard Rasch analysis. In this section, we also explain our approach to avoid exhaustive search on a survey with large numbers of items. Section 4 reports the results of the experiments on two artificial datasets (inhomogeneous and multi-dimensional surveys) and three publicly available real-world datasets. 


\section{Preliminaries}

\subsection{Generalized Partial Credit Model}

As explained in the introduction, in order to accomplish our goal we need to reevaluate our initial survey with $P$ items, on which we have responses from $N$ subjects, to a smaller and clinimetrically improved questionnaire. We consider the general case in which the responses are recorded in two or more ordered categories and write $x_{n i} \in\left\{0,1, \ldots, m_{i}\right\}$ for the observed response of subject $n$ on item $i$, where item $i$ consists of $m_{i}+1$ ordered categories. For binary responses, i.e., dichotomous test items, we simply set $m_{i}=1$. For polytomous test items we have $m_{i}>1$.

Rasch analysis relies on a mathematical, probabilistic model for the response of a subject on a particular item. In this paper, we will consider the so-called generalized partial credit model (GPCM), introduced in [28], a generalization of the original Rasch model to allow for polytomous test cases. This model contains different types of parameters. The parameter $\theta_{n}$ represents the ability of subject $n$ : the higher $\theta_{n}$, the larger the probability that subject $n$ gives high responses. We refer to the parameters $\beta_{i j}$, with $j=1, \ldots, m_{i}$ as the difficulties or thresholds of item $i$ : the higher $\beta_{i j}$, the higher $\theta_{n}$ needs to be to make a response larger than $j$ probable. In addition and unlike most standard approaches in Rasch analysis, the GPCM also includes so-called discrimination parameters $\alpha_{i}$ that model the predictability of the responses on item $i$.

According to the GPCM, the probability that subject $n$ on item $i$ gives response $x$ reads

$$
P\left(X_{n i}=x \mid \theta, \beta, \alpha\right)=\frac{\exp \left[\alpha_{i} \sum_{j=1}^{x}\left(\theta_{n}-\beta_{i j}\right)\right]}{1+\sum_{k=1}^{m_{i}} \exp \left[\alpha_{i} \sum_{j=1}^{k}\left(\theta_{n}-\beta_{i j}\right)\right]},
$$

for $x>0$, and

$$
P\left(X_{n i}=0 \mid \theta, \beta, \alpha\right)=\frac{1}{1+\sum_{k=1}^{m_{i}} \exp \left[\alpha_{i} \sum_{j=1}^{k}\left(\theta_{n}-\beta_{i j}\right)\right]}
$$

With $\alpha_{i}=1$ for $i=1, \ldots, P$, we obtain the (standard) partial credit model. In the dichotomous case, with $m_{i}=1$ for $i=1, \ldots, P$, the generalized partial credit model simplifies to the 2-parameter logistic model (2PL) and the standard partial credit model further simplifies to the original Rasch model [24, 26, 32].

\subsection{Penalized Joint Maximum Likelihood Estimation}

Given the observed responses $x_{n i}$, we then define the log likelihood for a particular set of items $\mathcal{S} \subset\{1, \ldots, P\}$ as

$$
L_{\mathcal{S}}(\theta, \beta, \alpha)=\sum_{i \in S} \sum_{n=1}^{N} \log P\left(X=x_{n i} \mid \theta, \beta, \alpha\right),
$$


with $P\left(X=x_{n i} \mid \theta, \beta, \alpha\right)$ from (1) and (2). This log likelihood is supposed to measure how well the parameters $\theta, \beta$, and $\alpha$ fit the subjects' observed responses on the items from set $\mathcal{S}$.

In this paper, we will apply (penalized) joint maximum likelihood estimation (JMLE) [21, 44, 46] whenever we aim to maximize this log likelihood. A well-known drawback of standard JMLE is its lack of convergence, especially when dealing with a perfect score or a completely zero score [2, 21]. Furthermore, the GPCM is nonidentifiable: its outcome does not change when we add a constant to all abilities and thresholds, nor when we multiply all abilities and thresholds with a constant and divide the discrimination parameters by the same constant. To solve these issues, we regularize the solutions by adding $L 2$ (ridge) penalty terms and define the penalized $\log$ likelihood

$$
F_{S}(\theta, \beta, \alpha)=L_{S}(\theta, \beta, \alpha)-\lambda_{\theta} \sum_{n=1}^{N} \theta_{n}^{2}-\lambda_{\alpha} \sum_{i \in S}\left(\ln \alpha_{i}\right)^{2} .
$$

We set a penalty on the (natural) logarithm of $\alpha_{i}$ to drive these discrimination parameters towards 1 , which would correspond to the standard partial credit model that typically underlies polytomous Rasch analysis. Similar, but slightly different penalties are considered by [29] for the 2PL model and, more recently, by [5] for the multidimensional item response theory (MIRT) model [33].

\section{The Proposed Method}

\subsection{In-plus-out-of-questionnaire Log Likelihood}

When designing an instrument, we keep items which help us to construct a clinimetrically optimal scale and drop the ones that do not which represent the clinical picture as part of our experts' point of view. We will refer to the corresponding sets as the included itemset $\mathcal{S}_{\text {in }}$ and the excluded itemset $\mathcal{S}_{\text {out }}=\{1, \ldots, P\} \backslash \mathcal{S}_{\text {in }}$, respectively. Our goal is to come up with a single criterion for judging the quality of any split into $\mathcal{S}_{\text {in }}$ and (thus) $\mathcal{S}_{\text {out }}$ based on the observed responses on the original survey. Given such a criterion, one's favorite optimization procedure can be applied to search for the included itemset $\mathcal{S}_{\text {in }}$ that maximizes it.

When we would indeed pick $\mathcal{S}_{\text {in }}$ as our final questionnaire, we can only make use of a subject's responses on these items to estimate her ability. To mimic this situation, we estimate the subjects' abilities that participated in the original survey only from their responses to $\mathcal{S}_{\text {in }}$, e.g., by maximizing the penalized log likelihood from (4):

$$
\left\{\hat{\theta}_{\mathcal{S}_{\text {in }}}, \hat{\beta}_{\mathcal{S}_{\text {in }}}, \hat{\alpha}_{\mathcal{S}_{\text {in }}}\right\}=\underset{\{\theta, \beta, \alpha\}}{\operatorname{argmax}} L_{\mathcal{S}_{\text {in }}}(\theta, \beta, \alpha)-\lambda_{\theta} \sum_{n=1}^{N} \theta_{n}^{2}-\lambda_{\text {in }} \sum_{i \in \mathcal{S}_{\text {in }}}\left(\ln \alpha_{i}\right)^{2} .
$$

We refer to the log likelihood

$$
\operatorname{IQ}-\operatorname{LL}\left(\mathcal{S}_{\text {in }}\right)=L_{\mathcal{S}_{\text {in }}}\left(\hat{\theta}_{\mathcal{S}_{\text {in }}}, \hat{\beta}_{\mathcal{S}_{\text {in }}}, \hat{\alpha}_{\mathcal{S}_{\text {in }}}\right)
$$

of these fitted parameters on the included itemset as the in-questionnaire log likelihood. We would like the in-questionnaire log likelihood to be relatively high and 
Algorithm 1 Recipe to compute the in-plus-out-of-questionnaire log likelihood for a particular included itemset $\mathcal{S}_{\text {in }}$ and a corresponding excluded itemset $\mathcal{S}_{\text {out }}$.

1. Fit abilities, thresholds, and discrimination parameters through penalized maximum likelihood estimation on the included itemset $\mathcal{S}_{\text {in }}$ as in (5).

2. Compute the in-questionnaire log likelihood of the parameters obtained in step 1 on the included itemset $\mathcal{S}_{\text {in }}$ as in (6).

3. Fit thresholds and discrimination parameters with the abilities fixed to those obtained in step 1 through (weakly) penalized maximum likelihood estimation on the excluded items $\mathcal{S}_{\text {out }}$ as in (7).

4. Compute the out-of-questionnaire log likelihood of the thresholds and discrimination parameters obtained in step 3 and the abilities obtained in step 1 on the excluded itemset $\mathcal{S}_{\text {out }}$ as in (8).

5. Add the out-of-questionnaire log likelihood from step 4 to the in-questionnaire log likelihood from step 2.

indeed, this is what typical test statistics in Rasch analysis like outfit and infit to some extent measure.

If, however, we trust that the designer of the original survey did a fine job and managed to come up with items that somehow relate to the latent construct that the final instrument is supposed to measure, the subjects' abilities $\hat{\theta}_{\mathcal{S}_{\text {in }}}$ should still provide a decent fit of the observed responses on the excluded set. To estimate the quality of this fit, we fix the abilities $\hat{\theta}_{\mathcal{S}_{\text {in }}}$ and only optimize over the thresholds and the discrimination parameters:

$$
\left\{\hat{\beta}_{\mathcal{S}_{\text {out }}}, \hat{\alpha}_{\mathcal{S}_{\text {out }}}\right\}=\underset{\{\beta, \alpha\}}{\operatorname{argmax}} L_{\mathcal{S}_{\text {out }}}\left(\hat{\theta}_{\mathcal{S}_{\text {in }}}, \beta, \alpha\right)-\lambda_{\text {out }} \sum_{i \in \mathcal{S}_{\text {out }}}\left(\ln \alpha_{i}\right)^{2} .
$$

We refer to the resulting

$$
\operatorname{OQ}-\operatorname{LL}\left(\mathcal{S}_{\text {out }}\right)=L_{\mathcal{S}_{\text {out }}}\left(\hat{\theta}_{\mathcal{S}_{\text {in }}}, \hat{\beta}_{\mathcal{S}_{\text {out }}}, \hat{\alpha}_{\mathcal{S}_{\text {out }}}\right)
$$

as the out-of-questionnaire log likelihood. Our final criterion, called in-plus-out-ofquestionnaire log likelihood (IPOQ-LL), adds the two log likelihoods together:

$$
\operatorname{IPOQ}-\mathrm{LL}\left(\mathcal{S}_{\text {in }}, \mathcal{S}_{\text {out }}\right)=\operatorname{IQ}-\mathrm{LL}\left(\mathcal{S}_{\text {in }}\right)+\operatorname{OQ}-\operatorname{LL}\left(\mathcal{S}_{\text {out }}\right) \text {. }
$$

Algorithm 1 summarizes the steps involved in the computation of the in-plus-outof-questionnaire log likelihood. The ipoq-ll is nicely balanced in the sense that every item in the original full survey always contributes once, either to the in-questionnaire or to the out-of-questionnaire log likelihood.

In all our experiments, we set $\lambda_{\theta}$ to 0.05 and $\lambda_{\text {in }}$ to 50 for the included itemset and $\lambda_{\text {out }}$ to 0.05 for the excluded itemset. Our procedure is largely insensitive to the precise setting of these parameters: just a little bit of regularization is sufficient to resolve issues with non-convergence and non-identifiability. The stronger regularization on the discrimination parameters in the included set and weaker (or even absent) regularization on the discrimination parameters in the excluded set is essential to stay close in spirit to standard Rasch analysis. 


\subsection{Comparison to Standard Rasch Analysis}

Standard Rasch analysis follows a manual iterative approach, in which items are evaluated and removed one by one if needed [14, 15, 40]. Typical evaluation criteria are item goodness-of-fit [45], unidimensionality [10, 13], and local dependency [8]. In the following, we will argue why we expect our procedure to naturally incorporate these criteria.

Goodness-of-fit. A standard procedure in Rasch analysis is to remove items with Infit and Outfit values that are very different from 1, and in particular those that are much larger than 1 (indicating more noise than expected). Our procedure will have a tendency to put predictive items in the included itemset: they help to reliably estimate the abilities and hence to achieve a higher log likelihood, not only on the included items but also on the excluded items. Too predictable or too unpredictable items are more likely to end up in the excluded set, with more flexibility (less penalty) to estimate the discrimination parameter.

Unidimensionality. Rasch analysis assumes that there is a single, unidimensional latent construct. Tests, such as the Martin-Löf test [7, 10], confirmatory factor analysis (CFA) [1, 13, 35, or principal component analysis (PCA) of the residual [6, 40, 42] can be used to confirm unidimensionality. As we will also see in the experiments, our procedure has an intrinsic drive to favor items belonging to a single dimension in the included set: including more of the same dimension helps to more reliably estimate the abilities corresponding to that dimension. The larger flexibility in fitting discrimination parameters attracts items that model different dimensions to the excluded set.

Local independence. The items that are put to Rasch analysis are assumed to be independent of each other. Dependent items may inflate scores and force the final scale's score in a particular direction [41] thereby increasing the risk of false positive or false negative results. Standard procedure is to keep just one of two items with highly correlated residuals. Also here, we expect our procedure to perform relatively well automatically: reliable estimation of abilities fares better from items with uncorrelated residuals than with correlated residuals.

\subsection{Stopping criterion}

The decision when the optimal combination of items to fulfill the Rasch model requirements has been achieved, can be arbitrary and is at least in part based on the subjective opinion of the Rasch modeler [3, 34]. Our procedure does provide an objective stopping criterion, namely at the maximum of IPOQ-LL. With the same regularization for the discrimination parameters, $\lambda_{\text {in }}=\lambda_{\text {out }}$, moving any item from the excluded to the included itemset will never decrease the IPOQ-LL. When choosing $\lambda_{\text {in }} \gg \lambda_{\text {out }}$, as we propose, there are two counteracting effects. On the one hand, moving an item from the excluded to the included itemset tends to increase the IPOQ-LL: it allows the abilities to adapt themselves to this item as well, increasing its corresponding log likelihood contribution. On the other hand, items in 
the excluded itemset enjoy more flexibility in estimating the discrimination parameter, which makes that items that are best modeled with a discrimination parameter quite different from 1 may fare better in the excluded itemset.

In our experiments (data not shown, but easily reproducible), we noticed that the size of the itemset that leads to the maximum IPOQ-LL is largely insensitive to the setting of the regularization parameters $\lambda_{\text {in }}$ and $\lambda_{\text {out }}$ as long as $\lambda_{\text {in }} \gg 1$ (strong regularization) and $\lambda_{\text {out }} \ll 1$ (hardly any or even no regularization).

\subsection{Itemset Selection}

On top of our single criterion for the quality of any split into an included itemset $\mathcal{S}_{\text {in }}$ and a corresponding excluded itemset $\mathcal{S}_{\text {out }}$, we can now use any optimization procedure to determine which items to keep. In fact, almost any approach originally designed for feature subset selection can be applied here as well [4, 9].

For a small number of items $P$ in the original survey, exhaustive search, going through all $2^{P}-1$ combinations, may still be feasible. Alternatively, we may want to first fix the size of the final questionnaire to $\left|\mathcal{S}_{\text {in }}\right|$ and then enumerate all $\left(\begin{array}{c}\left|\mathcal{S}_{\text {in }}\right| \\ P\end{array}\right)$ possible itemsets of that size.

For larger numbers of items in the original survey, exhaustive search is computationally too expensive and greedy approaches, such as backward elimination and forward selection, can be applied. Backward elimination is the search method of choice in standard Rasch analysis: starting from the full set of items, items are sequentially eliminated. Forward selection is the obvious alternative: starting from the empty set, items are sequentially added. In our experiments, we go for stepwise selection, which alternates between backward elimination and forward selection. Pseudocode for stepwise selection can be found in Algorithm 2.

Starting from a full itemset, OneStepBackwardElimination $\left(\mathcal{S}_{\text {in }}\right)$ in line 5 considers all possible $\left|\mathcal{S}_{\text {in }}\right|$ itemsets with one item less and returns the highest in-plus-outof-questionnaire log likelihood as well as the itemset that leads to this maximum. OneStepForwardSelection $\left(\mathcal{S}_{\text {in }}\right)$ in lines 8 and 11 does the same, but then by taking the best of all potential itemsets that can be constructed by adding one extra item to $\mathcal{S}_{\text {in }}$. The forward selection steps give the search procedure the possibility to (partially) recover when backward elimination too greedily excluded items that later in the process, when less items are left over, may turn out to be valuable after all.

\section{Experimental Study}

To evaluate our method, we conducted experiments on two artificial datasets (inhomogeneous and multi-dimensional surveys) and three publicly available realworld datasets. All experiments were run on a Dell PowerEdge R920, 4 x Xeon E7-4870v2 15C 2.3 GHz processor, 3072 GB RAM. We applied parallelization for the backward elimination function (Algorithm 2, line 5) and the forward selection function (Algorithm 2, line 8 and 11).

\subsection{Application to Artificial Data}

The artificial data experiments are meant to show that our semi-automated algorithm and the standard Rasch analysis apply similar procedures for removing items, 


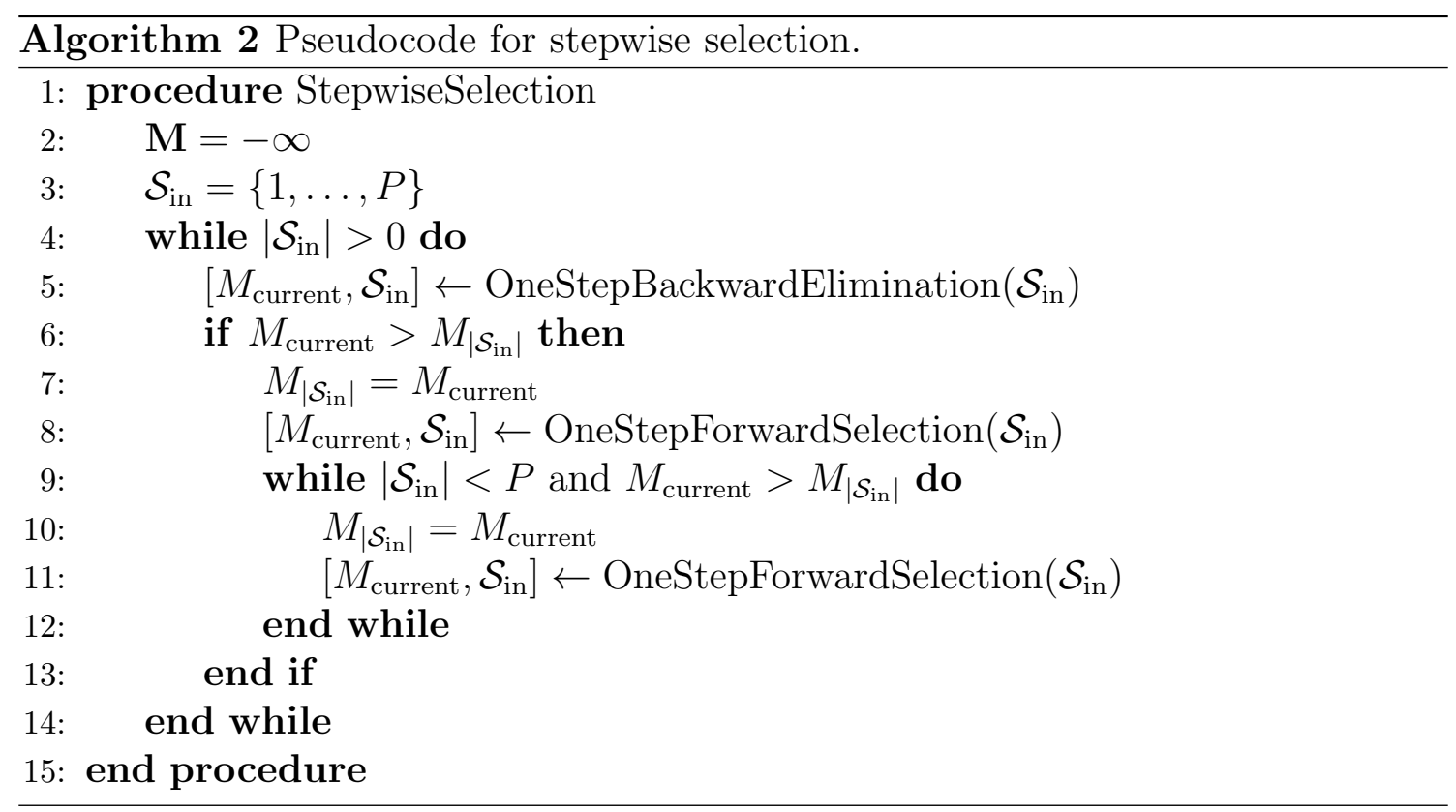

e.g., removing items which are relatively hard to predict and favoring items that belong to the same dimension. In these experiments, we consider two types of artificial surveys, that we refer to as inhomogeneous and multi-dimensional, respectively. For both types, we create surveys with either dichotomous or polytomous (with five categories) responses. All surveys have 18 items, subdivided into 3 subsets of 6 each, and 301 subjects. Responses are generated independently from the Generalized Partial Credit Model in (1) and (2), with $m_{i}=2$ for the dichotomous case and $m_{i}=5$ for the polytomous case, and parameter settings as further detailed in Table A1.

\subsubsection{Inhomogeneous Survey}

Responses in the inhomogeneous survey are generated with different discrimination parameters $\alpha \in\{0.002,0.02,2\}$ for each of the subsets. This makes the responses on items inhomogeneous as items in the first subset are relatively hard to predict, in the third subset relatively easy to predict, and in the second subset somewhere in between. From the goodness-of-fit criteria, we expect a Rasch analysis to first remove the items of the first subset, then the second subset, and finally the third subset.

Figure 1 displays Outfit and Infit values typically used in Rasch analysis against discrimination parameter values obtained by fitting a (Generalized) Partial Credit Model to the simulated data which reveals a strong relationship between those values. This relationship applies to both the dichotomous and the polytomous case.

Standard Rasch analysis tends to first remove the items with the highest Infit and Outfit values, which are those for which the responses are hardest to predict. As can be seen in Figures 2(a) and 2(c), for dichotomous and polytomous test items respectively, our semi-automated algorithm does the same for reasons explained in the Section 3.2 under Goodness-of-fit: everything else being the same, the IPOQ-LL score prefers items with lower discrimination parameters to be moved from the included to the excluded itemset. The maximum of the IPOQ-LL score as a function of the number of items in the included itemset in these simulations is obtained when 


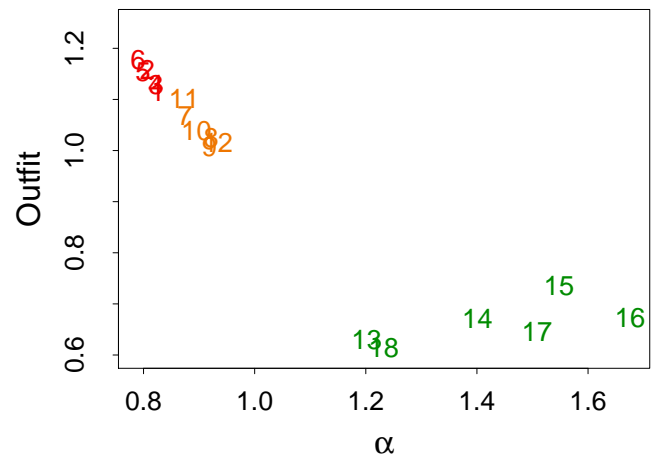

(a)

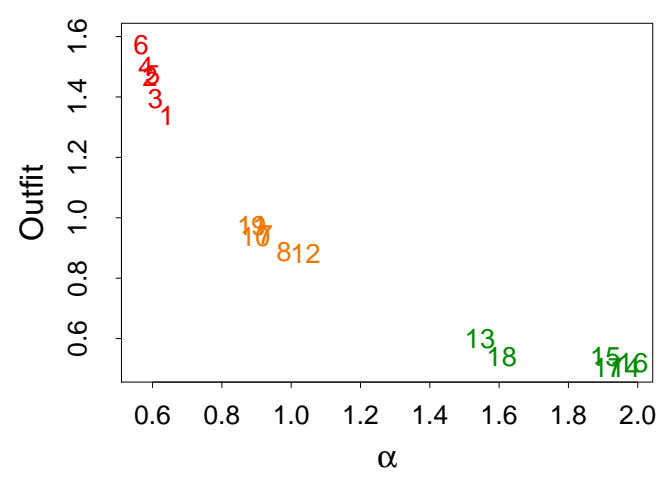

(c)

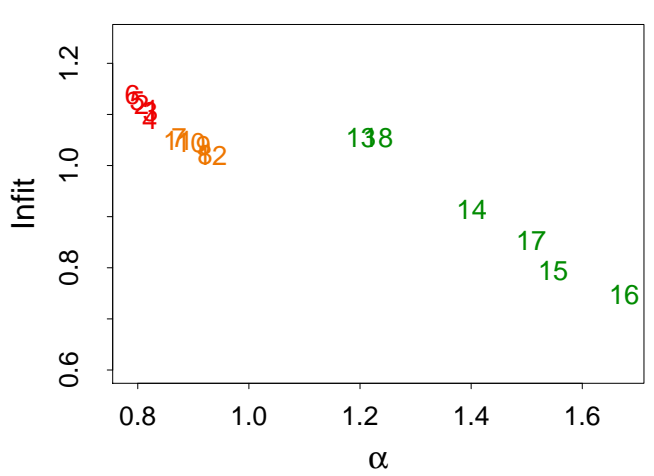

(b)

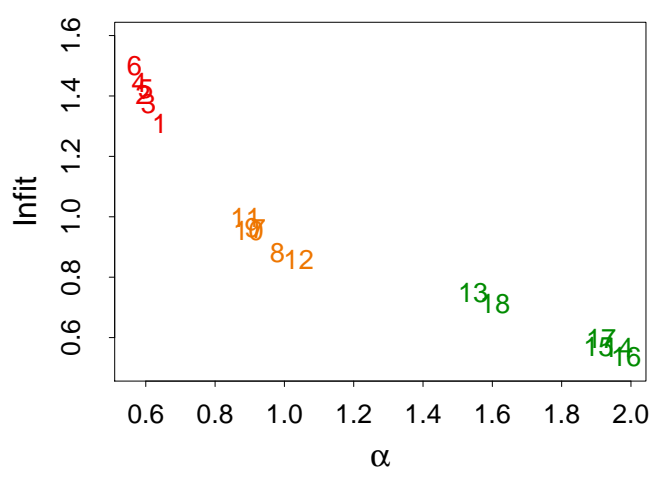

(d)

Figure 1: Discrimination parameters $(\alpha)$ against Outfit and Infit of the inhomogeneous survey case. (a) $\alpha$-Outfit plot of the dichotomous test items, (b) $\alpha$-Infit plot of the dichotomous test items, (c) $\alpha$-Outfit plot of the polytomous test items, and (d) $\alpha$-Infit plot of the polytomous test items.

the six most predictive items are still included. In practice, this may fluctuate a bit, also depending on the choice of the regularization parameters $\lambda_{\text {in }}$ and $\lambda_{\text {out }}$.

\subsubsection{Multi-dimensional Survey}

The multi-dimensional survey represents a situation in which each group of items corresponds to a different dimension. To achieve this, we choose subjects' abilities $\theta_{n}=-3, \ldots, 3$ for the first subset of items and randomly permute these for the two other subsets. The discrimination parameters for all items are the same. Which of the subsets is easiest to predict is now arbitrary, but we do expect a Rasch analysis to first remove all items from one subset, then from another subset, and only then from the remaining subset of items.

Figures 2(b) and 2(d), for dichotomous and polytomous test items, respectively, show that this is indeed what happens when applying our semi-automated procedure to the multi-dimensional survey: the algorithm removes one item and then continues to remove items belonging to the same subset (i.e., the same dimension), before removing any item from one of the other dimensions. This is in line with our argumentation in the previous Section 3.2 under Unidimensionality. Which dimension is preserved in these simulations depends on the surveys' responses and cannot be argued a priori as for the inhomogeneous survey. Again, the maximum of the IPOQ-LL occurs when six items are still included, but may slightly vary across simulations and for different choices of the regularization parameters. 


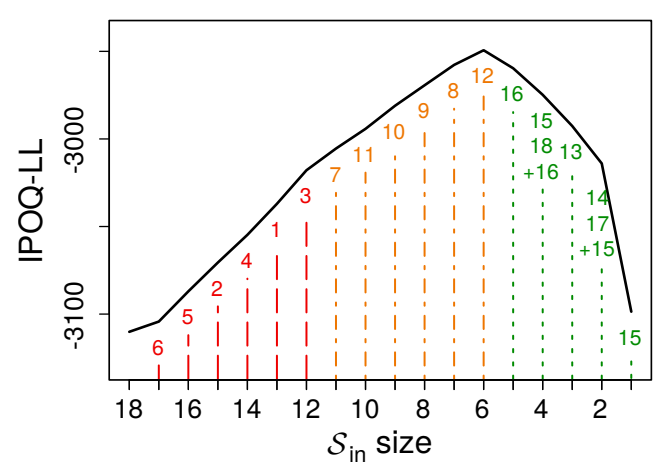

(a)

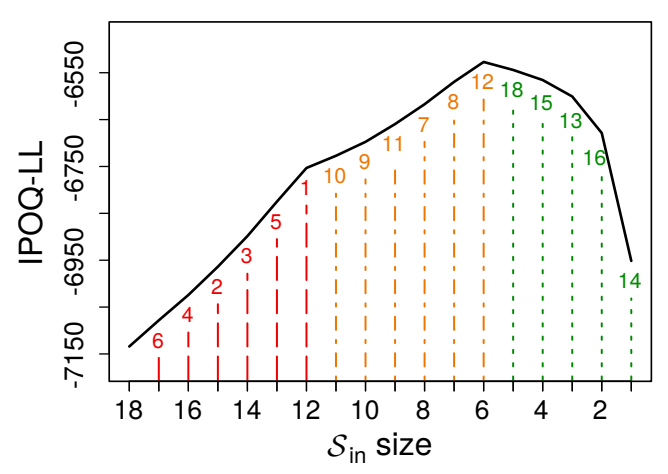

(c)

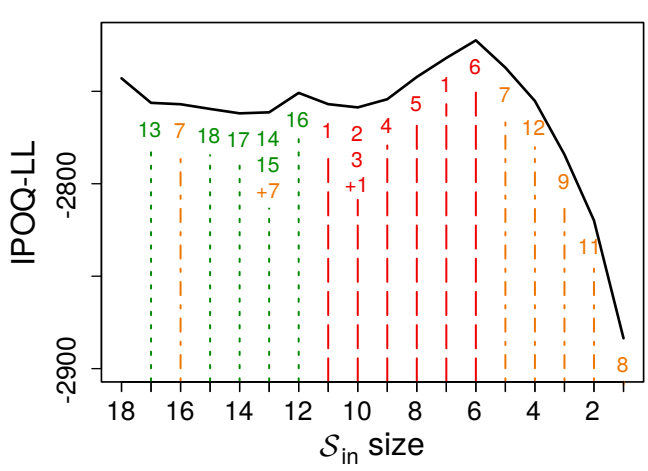

(b)

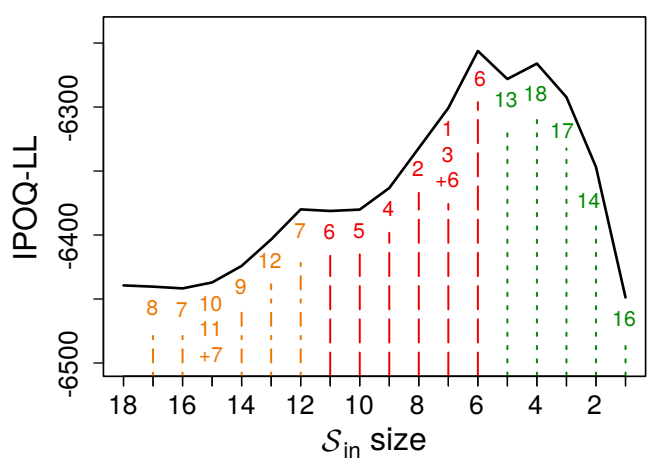

(d)

Figure 2: The highest IPOQ-LL score in different number of $\mathcal{S}_{\text {in }}$ for (a) dichotomous inhomogeneous survey (b) dichotomous multi-dimensional survey (c) polytomous inhomogeneous survey (d) polytomous multi-dimensional survey. The numbers on the plot shows in which order the items are removed, e.g., in (b) $\mathcal{S}_{\text {in }}$ size 13 was formed after removing items 14 and 15, but reintroducing item 7 which had been removed at the beginning.

\subsection{Application to Real-World Datasets}

For validation of our method on real-world data, we searched for datasets that satisfy the following criteria.

- The original dataset (survey with responses) is publicly available.

- A manual Rasch analysis has been applied to develop an instrument.

- The final instrument contains no differential item functioning or other special features.

- None of the authors of the current paper has been involved in the development of the instrument.

- The corresponding publication is less than 5 years old.

We found three such datasets: the sleep quality and distress inventory dataset [27], the trypophobia questionnaire dataset [18], and the coping health inventory for parents (CHIP) instrument dataset [11]. 


\subsubsection{The Sleep Quality and Distress Inventory Dataset}

The sleep quality and distress inventory is an instrument that was developed to measure the effect of sleep impairment on emotional distress in patients with various sleep disorders [27]. The original survey consists of responses from 457 subjects to 36 polytomous questions with four categories, i.e., "never", "sometimes", "often", and "always" which are coded as 1 to 4, respectively [27]. Since the subjects hardly choose categories 3 ("often") and 4 ("always"), these categories were then combined into a new category 3, "frequently" [27]. Applying manual Rasch analysis, Morrone et al. 27] ended up with 17 items as the final instrument. We will refer to this set of 17 items as the manual instrument. Using the same combination of categories, our semi-automated procedure leads to the result shown in Figure 3. Running the whole stepwise procedure on this dataset with 36 items takes about 20 minutes. The maximum of the IPOQ-LL occurs when 26 items are still included.

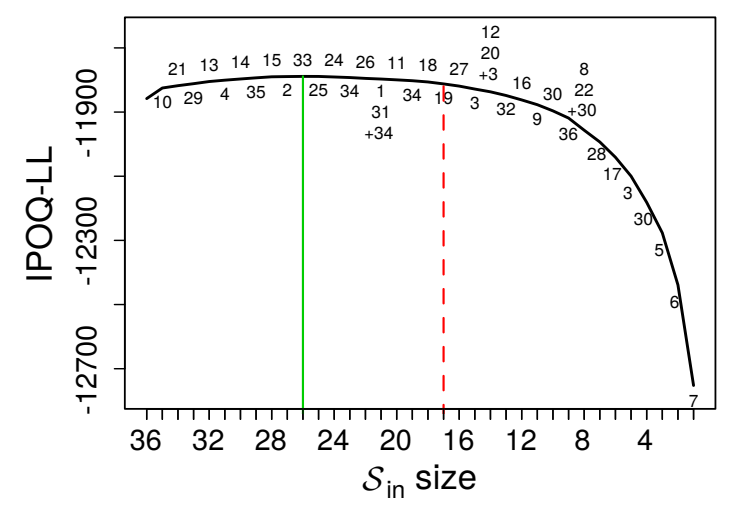

Figure 3: The highest IPOQ-LL score obtained for each number of included items $\left|\mathcal{S}_{\text {in }}\right|$ when running the semi-automated procedure on the sleep quality and distress inventory dataset. The solid green line indicates the highest IPOQ-LL score, which is obtained when 26 items are included. The dashed red line corresponds to the number of included items (17) in the original instrument, obtained through a manual Rasch procedure [27. The numbers on the plot tell in which order the items are removed, e.g., $\mathcal{S}_{\text {in }}$ of size 21 was formed after removing items 1 and 31, but reintroducing item 34 which had been removed before.

To prevent having to compare the 26 items from the semi-automated procedure against the 17 items in the manual instrument, for the semi-automated procedure we consider only the 17 items that correspond to the highest IPOQ-LL when 17 items are still included $\left(\left|\mathcal{S}_{\text {in }}\right|=17\right)$. We will refer to this set as the semi-automated instrument. The overlap between the semi-automated and the manual instrument is 14 items, which can be considered large: the probability of having an overlap of 14 or more items just by chance is smaller than $10^{-4}$. Items 24,31 , and 34 in the manual instrument are replaced by items 22, 27, and 36 in the semi-automated instrument (see also Table 2).

To further illustrate the clinimetric quality of both instruments, we consider standard Rasch statistics such as goodness-of-fit, local independence, unidimensionality, and reliability. For goodness-of-fit and local independence we take the mean over all items. For comparison we also compute these statistics for 10000 randomly drawn 17-item instruments.

Figures 4(a), 4(b), and 4(c) show histograms of some standard Rasch statistics 
for random instruments in which the statistics for the manual and semi-automated instrument are marked by red dashed and green solid lines, respectively. The mean Outfit and Infit values for both instruments are pretty close to 1, the recommended value, but the same applies to (most) random 17-item instruments. The residual correlation for the semi-automated instrument happens to be slightly lower than that for the manual instrument, but both are well within the acceptable range, and very comparable to those for random 17 -item instruments.

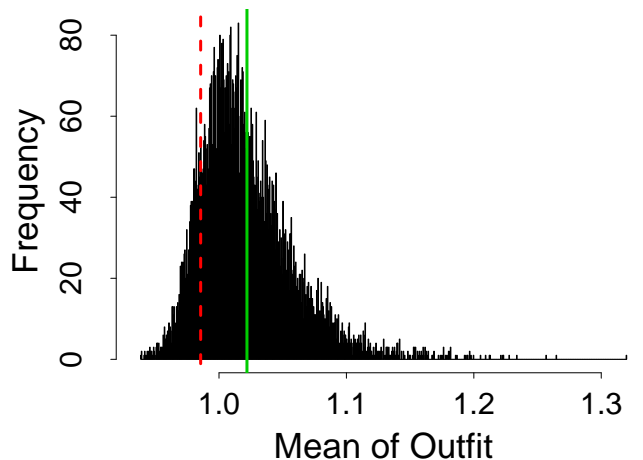

(a)

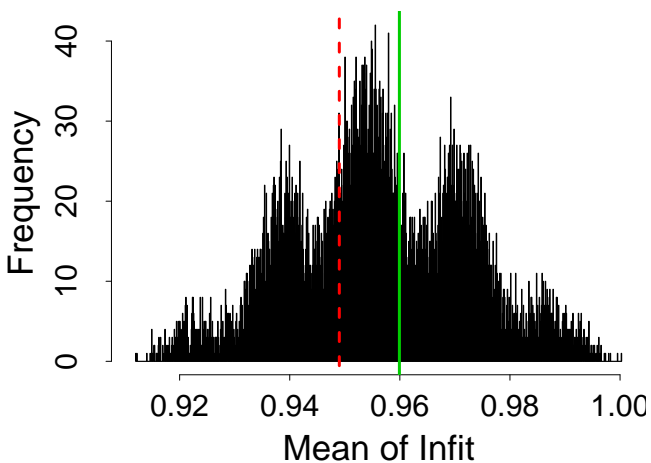

(b)

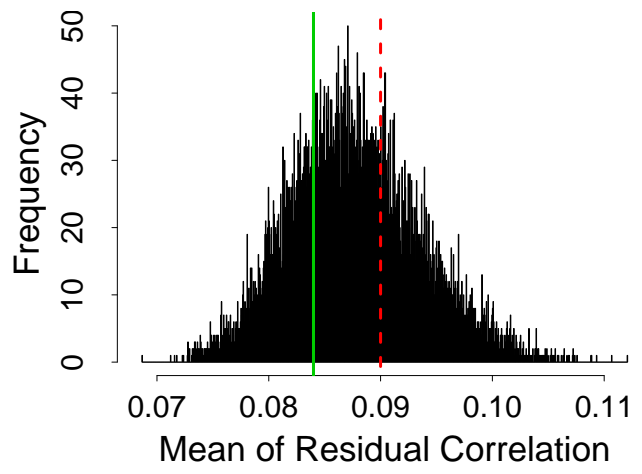

(c)

Figure 4: Statistics of the original 17-item instrument from [27] obtained through manual Rasch analysis (dashed red line), the optimal 17-item instrument according to the semi-automated procedure (solid green line), and 10000 random 17-item instruments (histogram) on the sleep quality and distress inventory dataset. (a) Mean of outfit MnSq. (b) Mean of infit MnSq. (c) Mean of residual correlation.

Table 1 compares some of the main Rasch statistics for the semi-automated and the manual instrument. The semi-automated instrument has a somewhat higher IPOQ-LL and lower mean residual correlation, where the manual instrument leads to a more narrow range of the Outfit and Infit Mean Square values per item.

Figures 5(a) and 5(b) show the Outfit and Infit values, respectively, of the 17 individual items in the semi-automated instrument as a function of the estimated discrimination parameters. As expected, both the Outfit and, in particular, the Infit values are strongly related to the discrimination parameters. Item 22 is the exception: it has a high Outfit value, yet relatively high discrimination parameter and relatively low Infit value. Looking more closely, the high Outfit value is solely due to a surprising response of a single subject (subject 220, see also Table 2). The high Outfit value likely explains why it did not make the final manual instrument. 
Table 1: High-level comparison between the original 17-item instrument from [27] obtained through manual Rasch analysis and the optimal 17-item instrument according to the semi-automated procedure.

\begin{tabular}{|c|c|c|c|c|}
\hline & IPOQ-LL & $\begin{array}{c}\text { Mean } \\
\text { Residual Correlation }\end{array}$ & $\begin{array}{c}\text { Range of } \\
\text { outfit MnSq }\end{array}$ & $\begin{array}{c}\text { Range of } \\
\text { infit MnSq }\end{array}$ \\
\hline Manual & -11837 & $\begin{array}{c}0.090 \\
\text { (no correlation }>0.3)\end{array}$ & $0.737-1.315$ & $0.758-1.282$ \\
\hline Semi-automated & -11812 & $\begin{array}{c}0.084 \\
\text { (no correlation }>0.3)\end{array}$ & $0.745-1.747$ & $0.570-1.307$ \\
\hline
\end{tabular}

This sensitivity of the Outfit statistic to just a few unexpected responses has been pointed out and warned about by Wright and Masters [45].

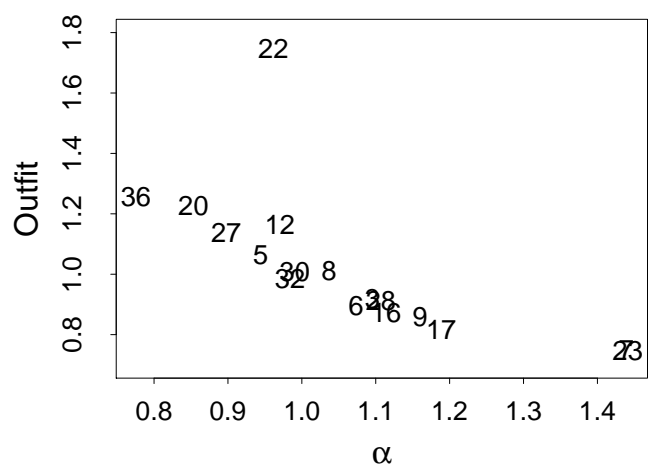

(a)

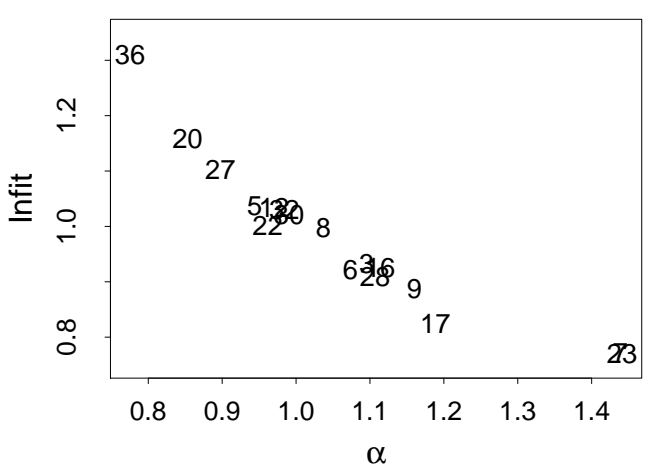

(b)

Figure 5: Estimated discrimination parameters $(\alpha)$ against the Outfit and Infit values of individual items in the semi-automated instrument for the sleep quality and distress dataset. (a) $\alpha$-Outfit and (b) $\alpha$-Infit.

To evaluate the unidimensionality of the instruments we use confirmatory factor analysis. Figure 6(a), 6(b), and 6(c) show the distribution of three commonly used fit indices, comparative fit index (CFI), Tucker-Lewis index (TLI), and root mean square error of approximation (RMSEA), respectively. For CFI and TLI, the higher the better, where values near to 1 indicate a close fit; for RMSEA, the lower the better. Both instruments do very well w.r.t. CFI and TLI and perfectly fine w.r.t. RMSEA, in all cases well within the acceptable ranges for good unidimensionality: $\mathrm{CFI}>0.95$, TLI $>0.95$, and RMSEA $<0.06$ [17].

A reliability measure that is commonly used in standard Rasch analysis is the person separation reliability (PSR) which indicates the overall performance of an instrument. It is the ratio of the true variance in the estimated measures to the observed variance and indicates the number of distinct person strata that can be distinguished. The commonly used threshold for this index is PSR $\geq 0.8$ [30]. As shown in Figure 7, both instruments, semi-automated and manual, excel in comparison with random instruments and have a good value of PSR, 0.864 and 0.866 , respectively.

Figure 8 compares both instruments using our own IPOQ-LL criterion. By definition, the semi-automated instrument is very well optimized for this criterion. 


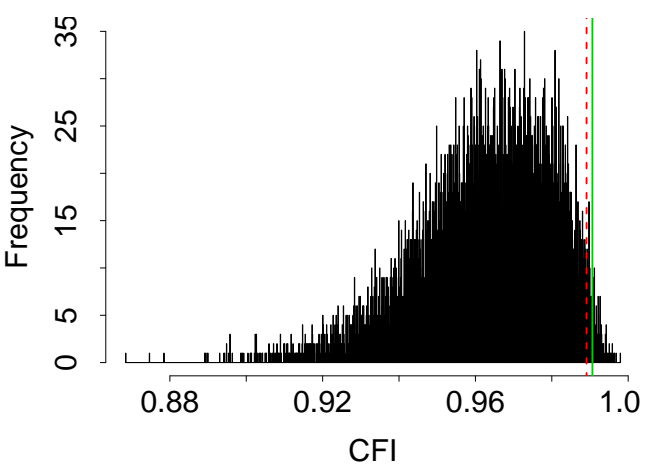

(a)

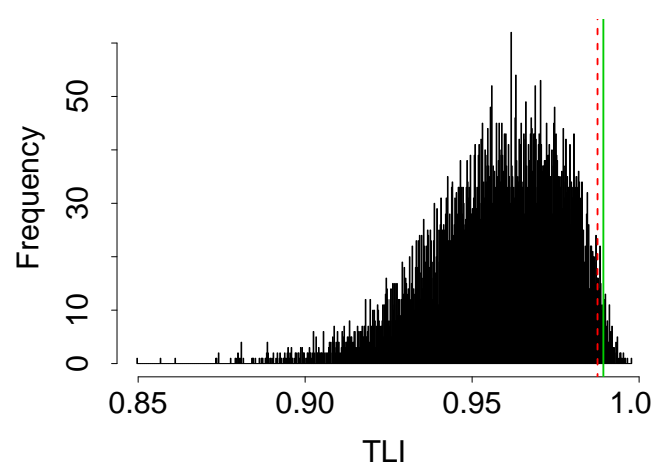

(b)

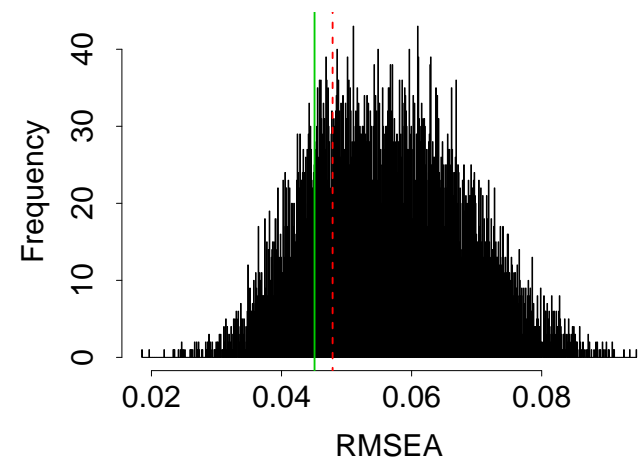

(c)

Figure 6: Unidimensionality test indices for the semi-automated instrument (solid green line), the manual instrument from [27] (dashed red line), and random 17-item instruments on the sleep quality and distress inventory dataset. (a) Comparative fit index (CFI) b) Tucker-Lewis index (TLI) (c) Root mean-square error of approximation (RMSEA).

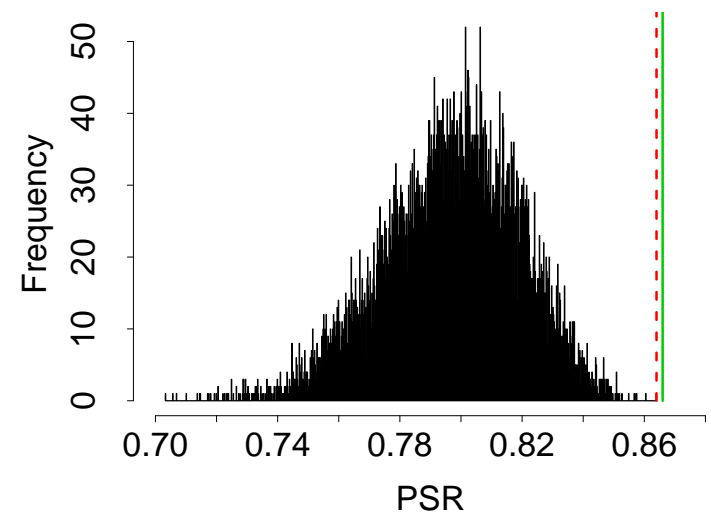

Figure 7: Person separation reliability (PSR) of the semi-automated instrument (solid green line), the manual instrument from [27] (dashed red line), and random 17-item instruments (histogram) on the sleep quality and distress inventory dataset.

The manual instrument does hardly worse and better than all randomly drawn 17item instruments, which appears to support our earlier argumentation that IPOQ-LL intrinsically captures many of the properties that a typical Rasch analysis cares about.

Considering the standard Rasch statistics, which are averages over all items and all subjects, we conclude that the manual and semi-automated instrument are 


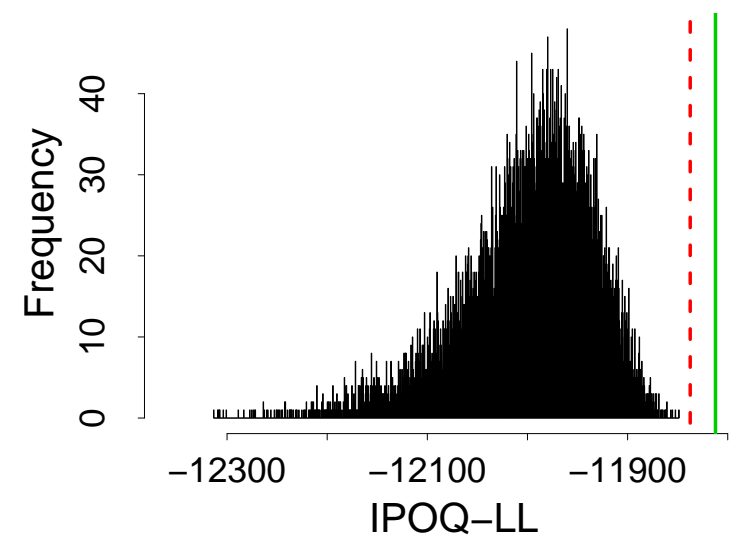

Figure 8: In-plus-out-questionnaire log likelihood (IPOQ-LL) values for the semi-automated instrument (solid green line), the manual instrument from [27] (dashed red line), and random 17-item instruments (histogram) on the sleep quality and distress inventory dataset.

Table 2: Responses of subjects 6,68 , and 240 on the items in the sleep quality and distress inventory dataset that differ between the semi-automated and manual instrument. For these subjects, it matters the most which instrument is used to estimate their abilities.

\begin{tabular}{|c|c|c|c|c|c|c|c|c|}
\hline \multirow{2}{*}{ Id } & \multicolumn{4}{|c|}{ Semi-automated } & \multicolumn{4}{c|}{ Manual } \\
\cline { 2 - 10 } & Item & Item & Item & Ability & Item & Item & Item & \multirow{2}{*}{ Ability } \\
& 22 & 27 & 36 & & 24 & 31 & 34 & \\
\hline 6 & 1 & 1 & 1 & -4.191 & 1 & 2 & 2 & -2.375 \\
\hline 68 & 1 & 1 & 1 & -4.191 & 1 & 2 & 1 & -3 \\
\hline 240 & 3 & 1 & 1 & -2.338 & 1 & 1 & 1 & -4.199 \\
\hline
\end{tabular}

clinimetrically very similar. We then also expect that the abilities estimated for individual subjects based on either the manual or the semi-automated instruments will be very much alike. Figure 9 plots these estimated ability parameters for the two instruments against each other. Indeed, the estimated ability parameters for the two instruments are highly correlated $(\rho=0.978)$.

For each estimated ability, we can also compute its standard error. The root mean squared standard error, 0.498 and 0.486 for the semi-automated and manual instrument, respectively, is visualized through the errorbars in the top left of Figure 9. For the subjects with id's 6, 68, and 240, corresponding to the three data points in the lower left corner away from the diagonal, the choice of instrument does appear to have a quite large effect on their estimated ability. Table 2 gives their responses on the non-overlapping items in the two instruments. Subjects 6 and 68 reported the lowest score on the items 22, 27, and 36 that are part of the semi-automated instrument, yet gave higher scores on items 24, 31, and 34 that are part of the manual instrument. This makes their "semi-automated ability" considerably lower than their "manual ability". More or less the opposite applies to subject 24, who (unexpectedly) gave the highest score on item 22 that is part of the semi-automated instrument, yet not of the manual instrument. 


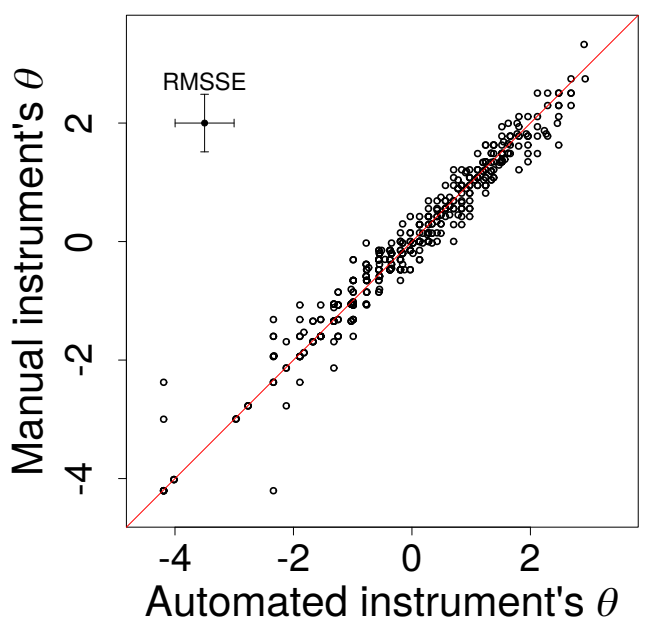

Figure 9: Estimated abilities for individual subjects based on the semi-automated against those based on the manual instrument. The root mean squared standard error for the estimates on both axes is visualized through the errorbars on the top left.

\subsubsection{Trypophobia Questionnaire Dataset}

The trypophobia questionnaire is an instrument that is developed to assess subjects' feelings and somatic responses toward clusters of roughly circular objects [18]. The original survey consists of responses from 582 subjects to 17 polytomous questions with five categories, i.e., "not at all", "slightly", "moderately", "considerably", and "extremely" which are coded as 1 to 5, respectively [18].

According to a standard Rasch analysis, the shortened version of the trypophobia questionnaire (with 14 items) has slightly better psychometric properties than the full version (with 17 items) [18]. Our semi-automated analysis of this dataset results in Figure 10. It took about 9 minutes to search along the 17 items. In this search, the maximal IPOQ-LL occurs when 16 items are still included, i.e., with only one item (number 9) removed. The itemset with 14 items that has the highest IPOQ-LL is identical to the original instrument obtained through a manual Rasch analysis.

\subsubsection{The Coping Health Inventory for Parents (CHIP) Dataset}

The CHIP instrument is an instrument which is used to measure the coping ability of parents having children with chronic disease [11. The original survey consists of 45 items divided into three subscales with 220 subjects. In this research, we only consider the first scale about "maintaining family integration, co-operation, and an optimistic definition of the situation", since Gothwal et al. 11] analyzed these scales separately using a standard Rasch analysis and found some hard to predict items in this scale.

The dataset of this first subscale consists of responses from 220 subjects to 19 polytomous questions with four categories, i.e., "not helpful", "minimally helpful", "moderately helpful", and "extremely helpful" which are coded as 0 to 3, respectively. However, subjects hardly chose category 2 ("moderately helpful") so it was combined with category 1 ("minimally helpful") into a single new category 1 called "somewhat helpful" [11]. Gothwal et al. [11] concluded that the revision of the first subscale has good psychometric properties after removing 4 items. With the same 


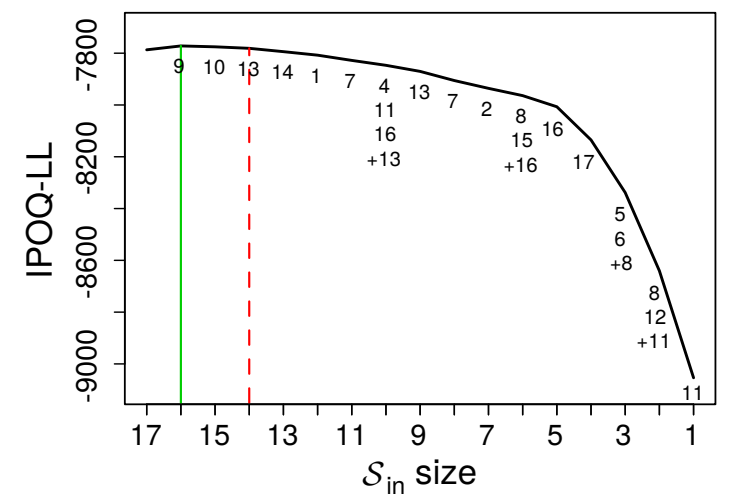

Figure 10: The highest IPOQ-LL score obtained for each number of included items $\left|\mathcal{S}_{\text {in }}\right|$ when running the semi-automated procedure on the trypophobia questionnaire dataset. The solid green line indicates the highest IPOQ-LL score, which is obtained when 16 items are included. The dashed red line corresponds to the number of included items (14) in the original instrument, obtained through a manual Rasch procedure [18. The numbers on the plot tell in which order the items are removed, e.g., $\mathcal{S}_{\text {in }}$ of size 6 was formed after removing items 8 and 15, but reintroducing item 16 which had been removed before.

setup, we applied our semi-automated procedure and obtained the result shown in Figure 11. It took about 4 minutes to search along the 19 items. In this search, the maximum of the IPOQ-LL occurs when 13 items are still included. Also for this survey, the semi-automated procedure returns the original instrument when we consider the itemset that has the maximal IPOQ-LL for the same number of items as the original instrument (15 in this case).

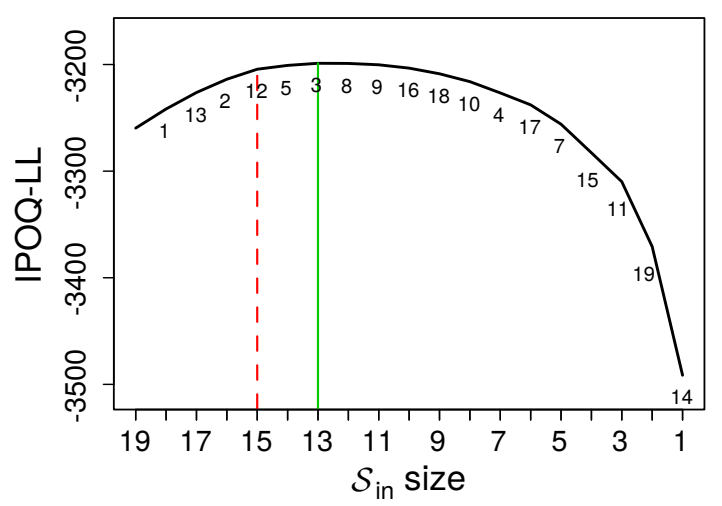

Figure 11: The highest IPOQ-LL score obtained for each number of included items $\left|\mathcal{S}_{\text {in }}\right|$ when running the semi-automated procedure on the first subscale of the CHIP dataset. The solid green line indicates the highest IPOQ-LL score, which is obtained when 13 items are included. The dashed red line corresponds to the number of included items (15) in the original instrument, obtained through a manual Rasch procedure [11. The numbers on the plot tell in which order the items are removed, e.g., $\mathcal{S}_{\text {in }}$ of size 15 was formed after removing item 12 .

\section{Discussion and Conclusion}

In this paper, we have described a novel procedure for semi-automated Rasch analysis. The procedure aims to optimize a new criterion, the so-called in-plus-outof-questionnaire log likelihood, IPOQ-LL. The philosophy behind this criterion is 
that a proper instrument should not just yield reliable estimates of a subject's scores on the items that are part of the instrument, but also (yet perhaps a bit less) on those that are left out. Through simulations and validations on real-world data, we have shown that our semi-automated procedure naturally incorporates desiderata for Rasch analysis related to goodness-of-fit and unidimensionality and leads to instruments that are very similar or even indistinguishable from those obtained with manual Rasch analysis (when constrained to the same number of items).

In our search for a working procedure that yields results similar to standard Rasch analyses, we noticed that two ingredients are essential: flexible discrimination parameters in the generalized partial credit model (instead of a standard Rasch model with all discrimination parameters set to 1) and stronger regularization of these discrimination parameters for the included items compared to the excluded items (instead of the same or no regularization for both). Without these ingredients, the procedure has a tendency to put items that are relatively difficult to predict in the included set, so that they can play a role in the construction of the scale. By providing more flexibility to the discrimination parameters in the excluded set, items that are difficult to predict can more easily get smaller discrimination parameters, so that incorrect predictions have a smaller impact on the log likelihood. The final outcome is largely insensitive to the setting of the regularization parameters involved: sensible default settings appear to work well for all simulated and realworld datasets considered in this paper.

A global criterion to measure the quality of any set of items, like the IPOQ-LL introduced in this paper, can be used in conjunction with any optimization approach to find the optimal set of items. Where standard Rasch analysis effectively applies backward elimination, removing items one by one, here we applied a stepwise procedure, also allowing for items to re-enter. At the expense of heavier computations, one can also go for (even) more involved optimization procedures such as hill climbing with restarts or evolutionary algorithms. However, it is doubtful whether calling in heavy optimization machinery really pays off in practice: even though simpler optimization approaches may fail to find the global optimum and hence the "best" instrument according to the precise optimization criterion, instruments corresponding to a local optimum may well be clinimetrically very similar. More generally, considering all the uncertainties involved, due to the finite number of subjects in any dataset, the vagueness of optimality criteria, and the arbitrariness in the applied statistical models, it hardly makes sense to claim that a procedure, be it manual or (semi-)automated, leads to an incontestable optimal instrument. As also suggested by the histograms in Figures 4(a) through 8 and the comparison between the manual and semi-automated instrument in Section 4.2.1, if a procedure finds one "optimal" instrument, there are likely many more that are virtually indistinguishable.

Our procedure can be extended in various ways, for example by adapting the underlying statistical model. An obvious and important extension would be the detection of differential item functioning (DIF), which occurs when individuals from different groups (e.g., male versus female) but with the same apparent ability systematically respond differently to a particular item. Many methods and procedures have been developed to detect DIF, see e.g., [16, 20, 23, 25, 37 39]. In our framework, DIF detection may be implemented by extending the generalized partial credit 
model to include a group-dependent term, e.g., along the lines of [36].

Another potential extension could be to try and derive multi-dimensional scales from a single dataset. On the multi-dimensional survey of Section 4.1.2, a greedy sequential approach will likely work: apply the semi-automated procedure to find an instrument, remove the corresponding items from the original dataset, and apply the same procedure once more to find the next instrument. A more involved integrated approach would adapt the generalized partial credit model to allow for multiple abilities per subject, corresponding to different dimensions, for example along the lines of [19, 22].

With or without extensions, we are careful to frame the procedure as semiautomated rather than fully automated. First of all, what comes out of the Rasch analyses, whether it is manual or semi-automated, will always depend on what goes in. So especially for creating a preliminary questionnaire and dataset, clinical expertise is required to ask the right questions. Furthermore, in subsequent analyses, one cannot do without sanity checks, much like in any statistical analysis. These include, for example, checks for under-utilized categories, re-scoring of items with disordered thresholds, as well as various after-run analyses. Given an appropriate original survey and accompanied with necessary sanity checks, our procedure has the potential to develop a valid, reliable, and clinimetrically robust instrument in a less time-consuming and more objective manner, thereby challenging the current practice of Rasch analyses and questioning the need for cumbersome manual procedures.

\section{References}

[1] Alexander, J., Vos, D., Brouwers, A., Schoot, T., Pat-El, R., Verboon, P., 2017. Early career burnout among Dutch nurses: A process captured in a Rasch model. Burnout Research 3, 55-62. doi:10.1016/j.burn.2016.06.001.

[2] Bertoli-Barsotti, L., 2005. On the existence and uniqueness of JML estimates for the partial credit model. Psychometrika 70, 517-531. doi:10.1007/ s11336-001-0917-0.

[3] Bond, T.G., Fox, C.M., 2015. Applying the Rasch Model: Fundamental Measurement in the Human Sciences. doi:10.1017/CB09781107415324.004, arXiv:arXiv:1011.1669v3.

[4] Cai, J., Luo, J., Wang, S., Yang, S., 2018. Feature selection in machine learning: A new perspective. Neurocomputing 300, 70-79. doi:10.1016/j.neucom. 2017. 11.077 .

[5] Chen, Y., Li, X., Zhang, S., 2018. Joint maximum likelihood estimation for high-dimensional exploratory item response analysis. arXiv preprint arXiv:1712.06748, 1-34arXiv: 1712.06748.

[6] Chou, Y.T., Wang, W.C., 2010. Checking dimensionality in item response models with principal component analysis on standardized residuals. Educational and Psychological Measurement 70, 717-731. doi:10.1177/0013164410379322. 
[7] Christensen, K.B., Bjorner, J.B., Kreiner, S., Petersen, J.H., 2002. Testing unidimensionality in polytomous Rasch models. Psychometrika 67, 563-574. doi:10.1007/BF02295131.

[8] Christensen, K.B., Kreiner, S., Mesbah, M., 2013. Rasch Models in Health. doi:10.1002/9781118574454.

[9] Derksen, S., Keselman, H.J., 1992. Backward, forward and stepwise automated subset selection algorithms: Frequency of obtaining authentic and noise variables. British Journal of Mathematical and Statistical Psychology 45, 265-282. doi:10.1111/j.2044-8317.1992.tb00992.x.

[10] Fischer, G.H., Molenaar, I.W., 1995. Rasch Models. doi:10.1007/ 978-1-4612-4230-7.

[11] Gothwal, V.K., Bharani, S., Reddy, S.P., 2015. Measuring coping in parents of children with disabilities: A Rasch model approach. PLoS ONE 10, 1-17. doi:10.1371/journal.pone.0118189.

[12] Gustafsson, J.E., 1980. Testing and obtaining fit of data to the Rasch model. Britsh Journal of Mathematical and Statistical Psychology 33, 205-233. doi:10. 1111/j.2044-8317.1980.tb00609.x.

[13] Hattie, J.A., 1985. Methodology review: Assessing unidimensionality of tests and items. Applied Psychological Measurement 9, 139-164. doi:10.1177/ 014662168500900204 .

[14] Hermans, M.C., Faber, C.G., De Baets, M.H., de Die-Smulders, C.E.M., Merkies, I.S.J., 2010. Rasch-built Myotonic Dystrophy Type 1 Activity and Participation Scale (DM1-Activ). Neuromuscular Disorders 20, 310-318. doi: $10.1016 / j . n m d .2010 .03 .010$.

[15] Hermans, M.C., Merkies, I.S., Laberge, L., Blom, E.W., Tennant, A., Faber, C.G., 2013. Fatigue and daytime sleepiness scale in myotonic dystrophy type 1. Muscle \& Nerve 47, 89-95. doi $10.1002 /$ mus.23478.

[16] Holland, P.W., Thayer, D.T., 1986. Differential item performance and the Mantel-Haenszel procedure. ETS Research Report Series 1986, i-24. doi 10 . 1002/j.2330-8516.1986.tb00186.x.

[17] Hu, L.T., Bentler, P.M., 2009. Cutoff criteria for fit indexes in covariance structure analysis: Conventional criteria versus new alternatives. Structural Equation Modeling 6, 1-55. doi:10.1080/10705519909540118.

[18] Imaizumi, S., Tanno, Y., 2018. Rasch analysis of the Trypophobia Questionnaire. BMC Research Notes 11, 1-5. doi:10.1186/s13104-018-3245-5.

[19] Kelderman, H., 1996. Multidimensional rasch models for partial-credit scoring. Applied Psychological Measurement 20, 155-168. doi:10.1177/ 014662169602000205 . 
[20] Komboz, B., Strobl, C., Zeileis, A., 2018. Tree-based global model tests for polytomous rasch models. Educational and Psychological Measurement 78, 128-166. doi:10.1177/0013164416664394.

[21] van der Linden, W.J., 2016. Handbook of Item Response Theory, Volume One: Models.

[22] Liu, Y., Magnus, B., O'Connor, H., Thissen, D., 2018. Multidimensional Item Response Theory. chapter 16. pp. 445-493. doi:10.1002/9781118489772.ch16.

[23] Lord, F.M., 2012. Applications of item response theory to practical testing problems. Routledge.

[24] Lord, F.M., Novick, M.R., 1968. Statistical Theories of Mental Test Scores.

[25] Magis, D., Tuerlinckx, F., 2015. Detection of differential item functioning using the Lasso approach. Journal of Educational and Behavioral Statistics 40, 111135. doi:10.3102/1076998614559747.

[26] Masters, G.N., 1982. A Rasch model for partial credit scoring. Psychometrika 47, 149-174. doi:10.1007/BF02296272, arXiv:arXiv:1011.1669v3.

[27] Morrone, E., Sguazzin, C., Bertolotti, G., Giordano, A., Braghiroli, A., Balestroni, G.L., Manni, R., Strambi, L.F., Castronovo, V., Zucconi, M., Carli, F.D., Pinna, E., Ottonello, M., Giorgi, I., Terzaghi, M., Marelli, S., Fanfulla, F., 2017. Development and validation of the Maugeri Sleep Quality and Distress Inventory ( MaSQuDI-17 ). PLoS ONE 12, 1-19. doi:10.1371/journal. pone.0180743.

[28] Muraki, E., 1992. A generalized partial credit model: Application of an EM algorithm. Applied Psychological Measurement 16. doi:10.1177/ 014662169201600206 .

[29] Paolino, J.P., 2013. Penalized joint maximum likelihood estimation applied to two parameter logistic item response models. Ph.D. thesis. Columbia University.

[30] Pesudovs, K., Burr, J.M., Harley, C., Elliott, D.B., 2007. The development, assessment, and selection of questionnaires. Optometry and Vision Science 84, 663-674. doi:10.1097/OPX.0b013e318141fe75.

[31] Rasch, G.G., 1966. An item analysis which takes individual differences into account. British Journal of Mathematical and Statistical Psychology 19, 4957.

[32] Rasch, G.G., 1980. Probabilistic Models for Some Intelligence and Attainment Tests.

[33] Reckase, M., 2009. Multidimensional Item Response Theory. New York, NY. doi:10.1007/978-0-387-89976-3. 
[34] Robinson, M., Johnson, A.M., Walton, D.M., MacDermid, J.C., 2019. A comparison of the polytomous Rasch analysis output of RUMM2030 and R (ltm/eRm/TAM/lordif). BMC Medical Research Methodology 19, 36. doi:10.1186/s12874-019-0680-5.

[35] Rosato, R., Testa, S., Bertolotto, A., Confalonieri, P., Patti, F., Lugaresi, A., Grasso, M.G., Toscano, A., Giordano, A., Solari, A., 2016. Development of a short version of MSQOL- 54 using factor analysis and item response theory. PLoS ONE 11, 1-15. doi:10.1371/journal.pone.0153466.

[36] Schauberger, G., Mair, P., 2019. A regularization approach for the detection of differential item functioning in generalized partial credit models. Behavior Research Methods , 1-16doi:10.3758/s13428-019-01224-2.

[37] Schauberger, G., Tutz, G., 2016. Detection of differential item functioning in Rasch models by boosting techniques. British Journal of Mathematical and Statistical Psychology 69, 80-103. doi:10.1111/bmsp.12060.

[38] Swaminathan, H., Rogers, H.J., 1990. Detecting differential item functioning using logistic regression procedures. Journal of Educational Measurement 27, 361-370. doi:10.1111/j.1745-3984.1990.tb00754.x.

[39] Tutz, G., Schauberger, G., 2015. A penalty approach to differential item functioning in Rasch models. Psychometrika 80, 21-43. doi:10.1007/ s11336-013-9377-6.

[40] Van Nes, S.I., Vanhoutte, E.K., Van Doorn, P.A., Hermans, M., Bakkers, M., Kuitwaard, K., Faber, C.G., Merkies, I., 2011. Rasch-built Overall Disability Scale (R-ODS) for immune-mediated peripheral neuropathies. Neurology 76, 337-345. doi:10.1212/WNL.0b013e318208824b.

[41] Vanhoutte, E.K., Hermans, M.C., Faber, C.G., Gorson, K.C., Merkies, I.S., Thonnard, J.L., 2015. Rasch-ionale for neurologists. Journal of the Peripheral Nervous System 20, 260-268. doi:10.1111/jns.12122.

[42] Vaughan, B., 2018. A Rasch analysis of the Revised Study Process Questionnaire in an Australian osteopathy student cohort. Studies in Educational Evaluation 56, 144-153. doi:10.1016/J.STUEDUC.2017.12.003.

[43] Wijayanto, F., Mul, K., Groot, P., van Engelen, B.G., Heskes, T., 2021. Semi-automated Rasch analysis using in-plus-out-of-questionnaire log likelihood. British Journal of Mathematical and Statistical Psychology 74, 313-339. doi: $10.1111 /$ bmsp. 12218 .

[44] Wright, B.D., Douglas, G.A., 1975. Best procedures for sample-free item analysis. Applied Psychological Measurement 14, 219-225.

[45] Wright, B.D., Masters, G.N., 1982. Rating Scale Analysis. Chicago.

[46] Wright, B.D., Panchapakesan, N., 1969. A procedure for sample-free item analysis. Educational and Psychological Measurement 29, 23-48. 
[47] Wright, B.D., Tennant, A., 1996. Sample Size Again. Rasch Measurement Transactions 9, 468.

[48] www.rasch.org, 2014. Rasch Measurement Analysis Software Directory. URL: https://www.rasch.org/software.htm. 


\section{Appendix A. Artificial Datasets}

This appendix describes how the artificial datasets for the experiments in Section 4.1 are generated. 


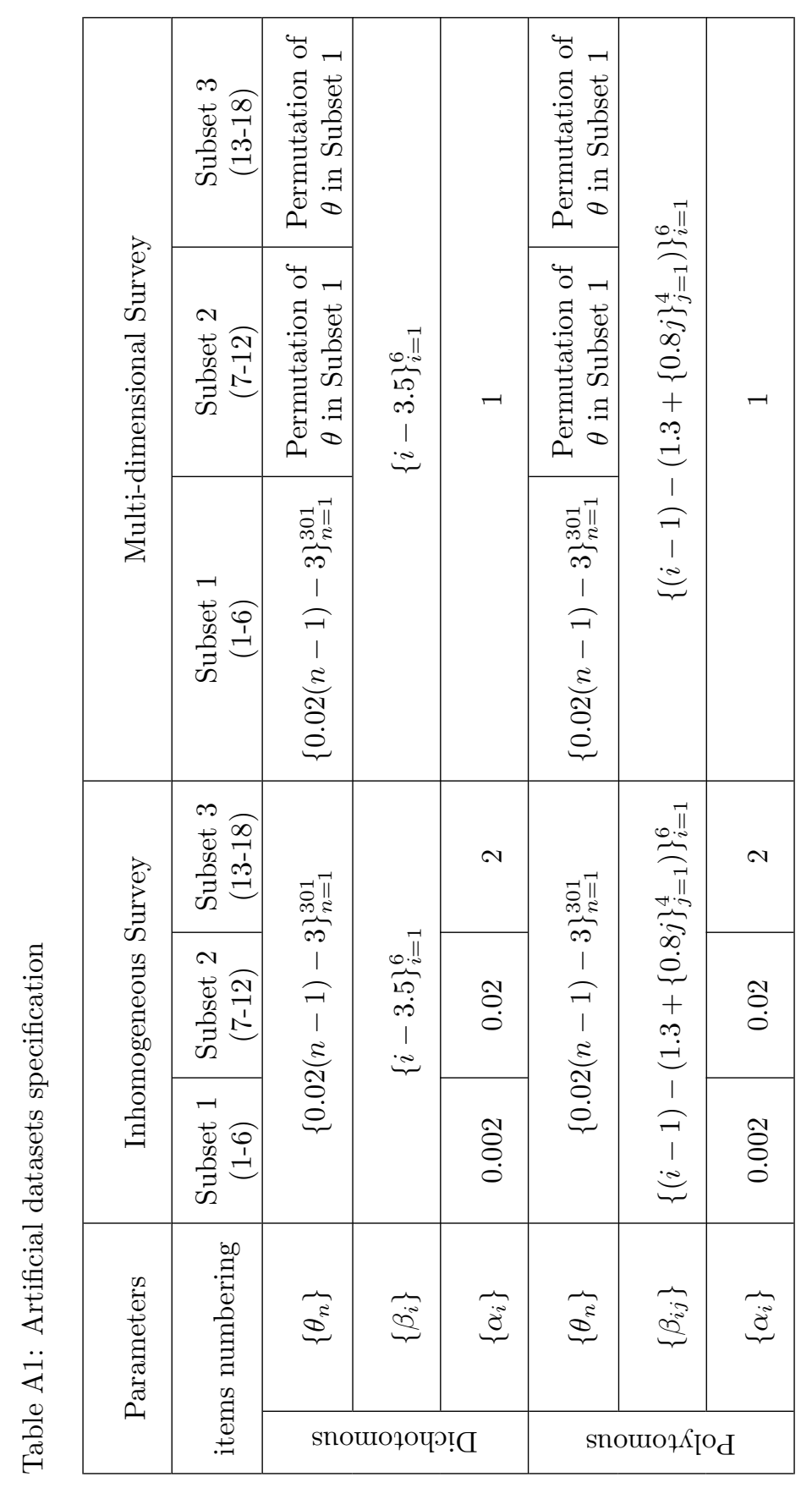

